\title{
Diffusion processes in multilayer transportation networks: the flight of the Coronavirus
}

\author{
A. Y. Yamamoto-Elizalde ${ }^{a, b}$, E. Hernández-Lemus ${ }^{b, c}$, and G. de Anda-Jáuregui ${ }^{b, d, *}$ \\ ${ }^{a}$ School of Sciences, National Autonomous University of Mexico (UNAM), México. \\ ${ }^{b}$ Computational Genomics Division, National Institute of Genomic Medicine, México. \\ ${ }^{c}$ Center for Complexity Sciences, National Autonomous University of Mexico (UNAM), México. \\ ${ }^{d}$ Programa de Cátedras CONACYT. National Council on Science and Technology (CONACYT), México. \\ *e-mail: gdeanda@inmegen.edu.mx
}

Received 28 February 2020; accepted 7 April 2020

\begin{abstract}
At the end of December of 2019, a new type of coronavirus, SARS-CoV-2, responsible of the disease now called COVID-19, started spreading in Wuhan, China and later throughout the world. Due to the global emergency state, the official pandemic declaration by the World Health Organization, as well as the need to investigate more about the danger Mexico is in, we worked on analyzing the risk of the COVID-19 importation to Mexico through the Air Transportation Network with a multilayer network approach. Based on the data obtained from the public data bases of OpenFlights, we created a multiplex network in which nodes represented airports, flights represented links, and airlines represented layers. We then simulated the propagation of the coronavirus using an unbiased random walk model with probability $p=1$ of infection once the random walker steps in a certain airport. We found the COVID-19 spread behavior the first month is anomalous (subdiffusion) and later behaves as a normal diffusion. We also found the risk of importing the virus to Mexico increases linearly over time and after approximately one year, there is almost a $90 \%$ probability of being infected. However, it is important to mention this high risk is due to contagions by people from other countries (not China) which have already confirmed cases of coronavirus. We concluded the risk of importing the COVID-19 to Mexico is almost ineludible over time unless effective medical interventions are imposed.
\end{abstract}

Keywords: COVID-19; random walk; diffusion; air transport network.

PACS: 02.10.0x; 89.90.+n

\section{Introduction}

At the end of December of 2019, some new cases of pneumonia were reported in Wuhan, China. It was later announced the pathogen was indentified as a new strain of coronavirus, $S A R S-C o V$-2, deriving in a disease named afterwards COVID-19, which was presumably originated at a market in Wuhan, the capital of the Hubei province [1]. At the end of January of 2020, people from different countries of Asia, Europe and North America had already been infected, others had died and numerous cases are still closely monitored [2]. As of April 1, 2020, 823,626 confirmed cases and 40,598 deaths have been reported [3].

Although there have been great efforts to treat the novel virus, thousands of people have lost their lives and there is still no cure available. The Chinese, as well as other countries' governments have tried to contain the infection by quarantining the citizens of Wuhan and other cities. However, the infection is still propagating resulting in the World Health Organization's (W.H.O.) declaration of Public Health Emergency of International Concern [4] and on March 11, COVID-19 was characterized as a pandemic [5].

This infection has raised concern for the rapid propagation and human-to-human contagion, transmitted through droplets of saliva or discharge from the nose when an infected person coughs or sneezes [6]. There was concern about the virus transmission as the Tokyo Olympic and Paralympic Games were less than six months away and that would have
DOI: https://doi.org/10.31349/RevMexFis.66.516

meant a huge movement of people through the Air Transportation Network (ATN). As a result, the Olympic Games were postponed until late July of 2021 [7]. Motivated by its quick spread between countries, we studied the diffusion of the COVID-19 through the ATN with a network science approach.

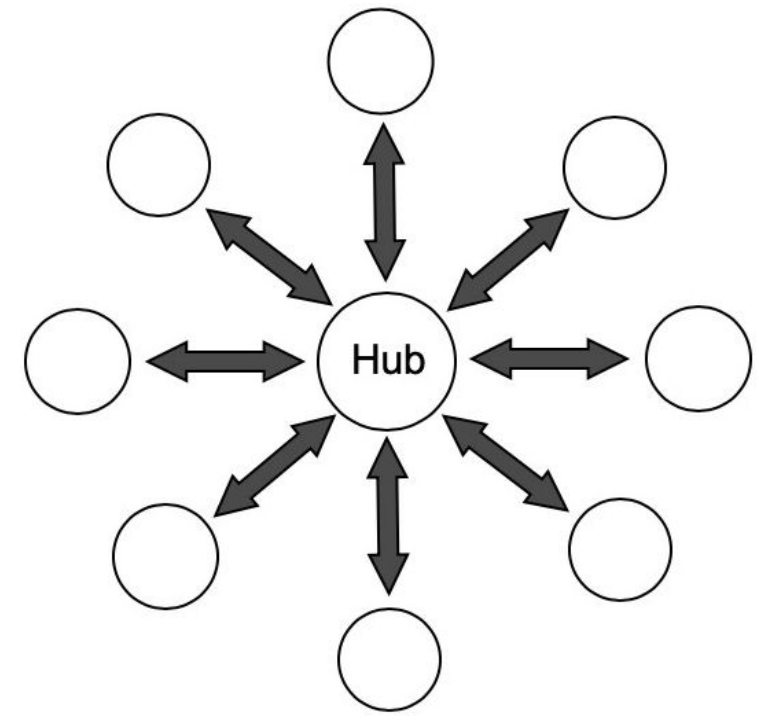

FIGURE 1. Diagram of the Air Transport Network preferred distribution known as spoke-hub. 
The ATN is one of the most important transport systems worldwide mainly because it connects almost all the world, with the exception of five countries which do not have an airport in their territory [8].

The organization the ATN primarily has is called spoke$h u b$, which is a system with a star-like network distribution [9,10]. In this model, important airports or hubs are used to concentrate flights towards other smaller airports as shown in Fig. 1. This type of distribution is preferred by the majority of airlines because it lets them offer more flights and maximize the conection to different airports while minimizing the numer of flights [9]. Although this distribution has great benefits, in cases like the one we are experiencing with the COVID-19, this model eases the disease propagation.

From a network perspective, the collection of airports and routes can represent a complex network. From this standpoint we can understand and analyze its connections and dynamic processes such as diffusion.

\subsection{Multilayer network formalism}

A network, mathematically known as a graph, is a collection of elements called nodes that are interacting with each other, therefore connected by edges.

The nodes and edges do not have to be of the same kind, such structures can be represented with a multilayer network. A multilayer network is a set of networks, each of which depicts a different type of interaction. Every one of these networks is called a layer.

A multilayer network, $M$, is defined as:

$$
M=(Y, G, \Gamma)
$$

where $Y$ defines the set of layers:

$$
Y=\{\alpha \mid \alpha \in 1,2, \ldots, M\}
$$

of the multilayer network and $M=|Y|$.

$G$ is the ordered list of networks that characterize the interactions in each one of the layers $\alpha=1,2, \ldots, M$, i.e.,

$$
G=\left(G_{1}, G_{2}, \ldots, G_{M}\right),
$$

where $G_{\alpha}=\left(V_{\alpha}, E_{\alpha}\right)$ is the network of the layer $\alpha$.

The layer's set of nodes $\alpha$ is denoted by $V_{\alpha}$ and the set of edges within this layer is $E_{\alpha}$ that are also known as intralinks.

Finally, $\Gamma_{\alpha, \beta}$ characterizes the interactions through the different layers $\alpha$ and $\beta$, known as interlinks.

A multiplex network is a particular case of a multilayer network with the following properties [11]:

1. Multiplex networks are multilayer networks in which there is a one-to-one mapping of the nodes in the different layers, known as replica nodes.

2. Interlayer links can only connect a node with its replica.

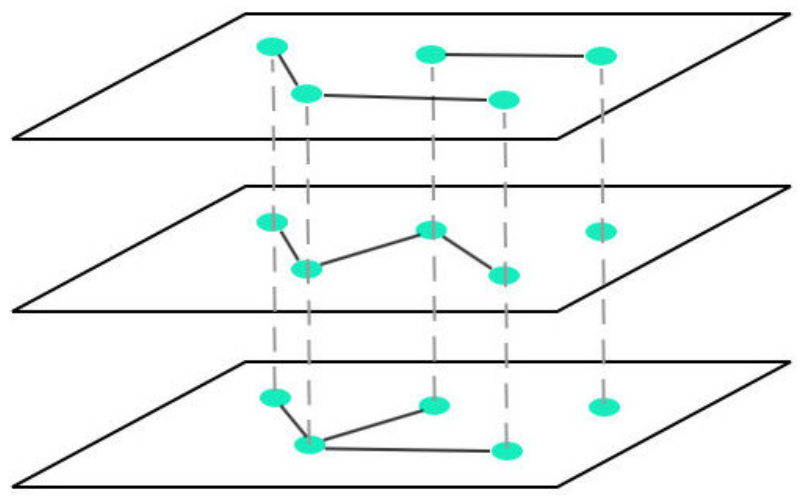

FIGURE 2. Diagram of a multiplex network. Each layer contains a different network that represents a certain type of interaction (intralinks). The dotted lines (interlinks) between the layers connect a node with its corresponding replica.

Because in a multiplex network, there is no explicit treatment of interlinks, it can be viewed as:

$$
M=(Y, G)
$$

where $G=\left(G_{1}, G_{2}, \ldots, G_{M}\right)$ and each network $G_{\alpha}=$ $\left(V_{\alpha}, E_{\alpha}\right)$ is formed by the same set of nodes:

$$
V_{\alpha}=V=\{i \mid i \in\{1,2, \ldots, N\}\}
$$

and by the set of links $E_{\alpha}$.

In this case, a multiplex network without interlinks has its whole information encode in a matrix called adjacency matrix, $a$ :

$$
a_{i j}^{[\alpha]}= \begin{cases}1 & \text { if nodes } i \text { and } j \text { interact in layer } \alpha \\ 0 & \text { otherwise }\end{cases}
$$

\subsection{Network epidemiology}

The propagation of infectuous diseases is one of the most studied dynamic processes. Epidemiology is the practice that focuses on studying health problems, their characteristics, distribution, and risks. As we mentioned before, we are interested in the propagation of the current outbreak of coronavirus SARS-CoV-2.

Diffusion of any kind, such as diseases, in a network is a fundamental dynamic process described by the transport of a continuous quantity along its edges [11]. There are several ways of modeling diffusion in a network, such as SIR and SIS models [12,13], nevertheless, one of the simplest forms is with a random walk model.

\subsection{Random walk model diffusion}

According to the model with an unbiased random walk, a particle located at node $i$ has the same probability of moving to any neighbor node $j$ of node $i$. This means, if node $i$ is connected to $k$ different nodes, the probability of the random walker to take a step from node $i$ to node $j$ is $p=1 / k$. 
This irregular movement or Brownian motion modeled by the random walk considers the mean square displacement. As stated by Frenkel et al. [13], considering an ensemble average, over the trajectories of $N$ particles, the mean square displacement at a time $t$ is defined as:

$$
\left\langle\Delta r_{i}^{2}(t)\right\rangle=\frac{1}{N} \sum_{i=1}^{N}\left|r_{i}(t)-r_{i}(0)\right|^{2},
$$

with $\Delta r$ the displacement of a Brownian particle in a given time interval $t$.

The mean square displacement considered in the brownian motion is given by the Einstein's relation [12]:

$$
\left\langle(\Delta r(t))^{2}\right\rangle=2 n D t,
$$

where $\Delta r$ is the displacement of a Brownian particle in a given time interval $t, n$ is the spatial dimension, and $D$ the diffusion coefficient.

Nonetheless, anomalous diffusion processes exist, i.e., those where the mean square displacement does not follow Eq. (8).

These types of anomalous diffusion follow [12]:

$$
\lim _{t \rightarrow \infty}\left\langle(\Delta r)^{2}\right\rangle \sim t^{\alpha},
$$

where $\alpha$ is a real positive number and $\alpha \neq 1$.

Specifically, we can have three cases: superdiffusion, normal diffusion, and subdiffusion, as summarized in Table I.

TABLE I. $\alpha$ conditions for the different types of diffusion.

\begin{tabular}{cc}
\hline Type of diffusion & $\alpha$ \\
\hline Superdiffusion & $1<\alpha$ \\
Normal diffusion & $\alpha=1$ \\
Subdiffusion & $0<\alpha<1$ \\
\hline
\end{tabular}

\section{Methodology}

The work flow we followed during this project is shown in Fig. 3. We first downloaded the airline routes and airports data bases from which we got the necessary information to construct the networks. Both data bases were acquired from OpenFlights, one of the largest and most complete public bases $[14,15]$.

The information of airports (updated in 2019) and routes (updated in 2017) was analyzed and filtered so that we only worked with active airports and routes without stops or codeshare operated flights.

Based on the remaining data, we constructed 29 networks of two different types: monoplex and multiplex. We worked with 4 networks of the latter type (the ATN and three alliances: Sky Team, Star Alliance, and oneworld), which correspond to those networks that consist of layers representing every airline in an alliance or in the whole ATN. The remaining 25 networks were monoplex, one for every airline that operates flights from the Wuhan airport. Every network is considered to be a set of nodes or airports with links between them if a direct flight connect them.

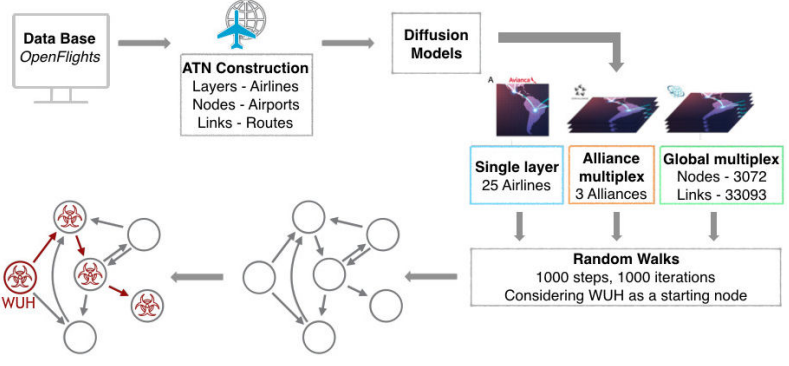

FIGURE 3. Diagram of the simulation work flow, starting with the acquisition and processing of the airports and routes data. The second step is the construction of the networks and then the diffusion simulation with an unbiased random walk is developed.

The division between the multiplex and monoplex networks was made to characterize the diffusion in different network topologies.

Once the networks were created, we simulated the virus propagation phenomenon with a model of random walks with a probability $p=1$ of contagion, i.e., if passed at least once through an arbitrary airport, this one would be considered infected.

Our model took into account the initial airport as "WUH", the IATA code for the airport located in Wuhan. The random walker took 1000 steps. Considering that a person is able to take three flights in a single day at most, these 1000 steps would correspond to approximately one year in our simulation. The diffusion simulation with the parameters mentioned above was repeated 1000 times to robust our statistics.

We considered as infected nodes the airports located at the cities in which confirmed cases existed as of January 26, 2020 to see if the COVID-19 importation to Mexico was through these airports. We did not take into account the travel ban within Wuhan nor within any other country. However, Chinazzi et. al [13] showed that despite this precautionary measure, the overall epidemic progression is only delayed by 3 to 5 days, so the results of our simulations will be almost the same as if we had taken the travel ban into account.

During the analysis of the COVID-19 importation to Mexico, we considered the list of 76 mexican airports. With that information we looked for the number of random walks, out of the 1000, that arrived at a mexican airport at some point in time and if the coronavirus reached Mexico from Wuhan or from any other place already infected.

The code of everything we did can be found in GitHub: https://github.com/Yuriyama/Coronavirus

\section{Results and Discussion}

The simulations resulted in a collection of 1000 random walks starting in Wuhan, China. With these simulations, we found that the overall infected portion of the ATN after approximately one year is $13.62 \%$. The ATN's infected area by the COVID-19, if only traveled through a single airline or 


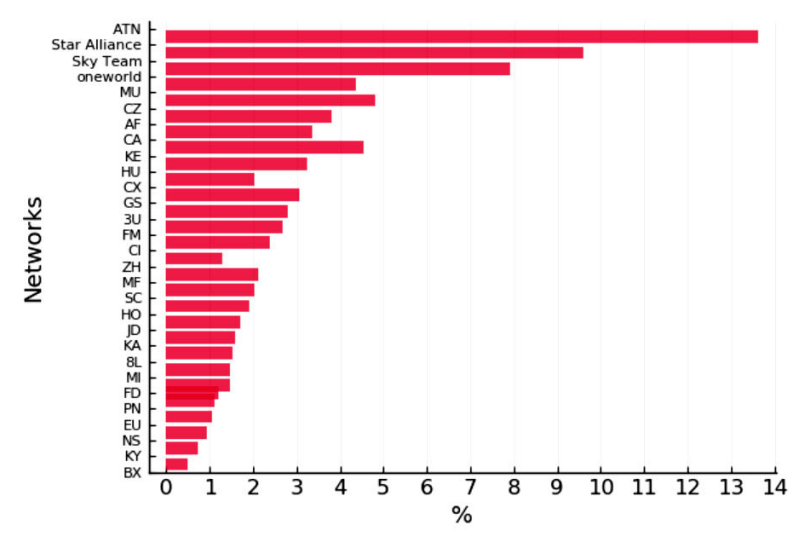

FIGURE 4. Histogram of the infected percentage of the Air Transportation Network if travelled through certain airlines or alliances after approximately one year. Networks appear from least number of airports (BX) to most (ATN).

or alliance is also shown in Fig. 4, where the percentage is detailed by network.

On the vertical axis of Fig. 4, the 29 networks studied are shown, from the least amount of airports in a network, corresponding to $\mathrm{BX}$, to the network containing the largest amount of airports, which is the ATN with 3072 airports and 33093 routes.

The general behavior shows that the more number of airports a network has, the bigger the infected area is. However, the network corresponding to CA (Air China) has a bigger area of contagion with respect to other airlines of approximately the same size node-wise possibly because it is the most important airline in China; thus having more routes from and to China.

In Fig. 4 is shown that after approximately one year, out of the 3072 airports that create the ATN, 418.34 airports are infected in average, corresponding to the $13.62 \%$ of the ATN.

To study the risk of importing the COVID-19 virus to Mexico, we focused only on those random walks that at some point reached a mexican airport.

We found that in about 210 flights through the ATN, the infection arrives at Mexico. Also, in all of our simulations, Mexico was first infected after passing through one or several already infected airports. None of them was due to a "direct" contact between Wuhan and Mexico. This confirms what Chinazzi et al., [13] concluded about the number of cases observed outside China would resume its growth after 2 or 3 weeks because of cases that originated elsewhere.

The risk of importing the COVID-19 to Mexico through different networks after a year is shown in Fig. 5. Not all of the 25 airline networks appear because no random walk reached mexican airports while travelling through those (23) networks.

From the monopolex approach, the only way for Mexico to get infected is through the Air France airline (AF) and Air China airline (CA). As we mentioned, the random walks through other monoplex airlines did not reach any mexican

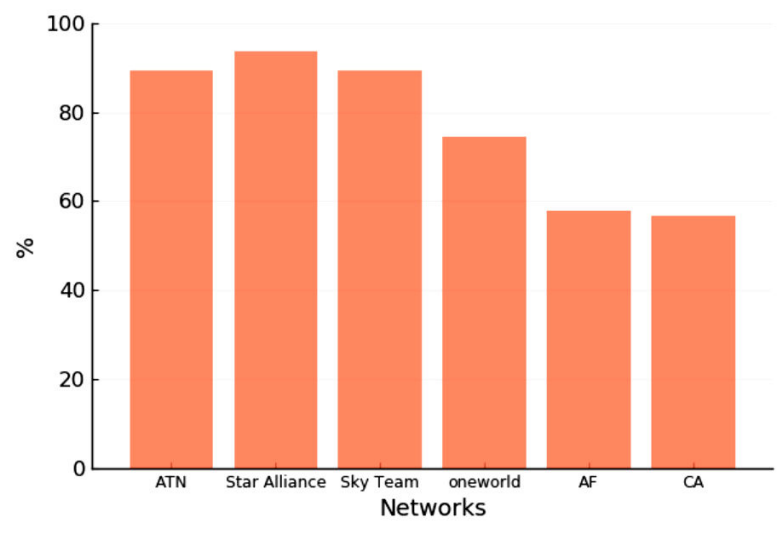

FIGURE 5. Amount of random walks that infect a mexican airport at any given time of the year simulation presented by network.

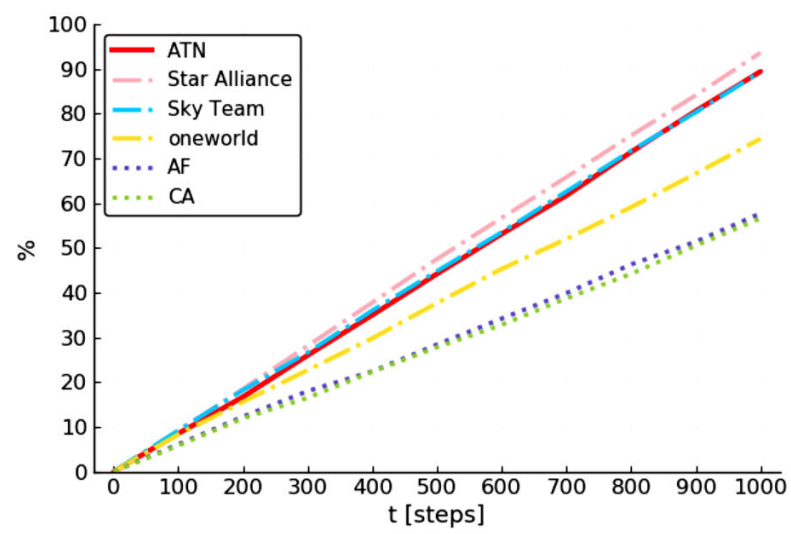

FIGURE 6. Risk or probability of importing the virus to any mexican airport as a function of time presented by network.

airport after one year of simulation. However, if we consider the whole ATN network, we got $89.4 \%$ of Mexico's airports infected. So we can predict that the infection will eventually reach Mexico.

If analyzed through time, the importation risk to any airport of Mexico increases linearly. This is shown in Fig. 6.

Figure 6 shows the importation risk of coronavirus to Mexico over time in the different networks. All the multiplex networks (ATN and alliances) begin with the same risk the first month. After the first month and a half, the risk changes between the alliances and monoplex networks. Star Alliance, Sky Team, and the ATN end with a higher probability than other networks. Still at the end of the simulated year, all of them represent a risk of more than $50 \%$ to Mexico.

At 500 steps or approximately five and a half months, the probability of importing the COVID-19 to any part of Mexico traveling through the ATN is of $44.2 \%$ and there is a $75 \%$ of risk after approximately 850 steps since the outbreak of the coronavirus which has been estimated in September.

From these results we can assume that the risk of importing the coronavirus to Mexico is almost certain as time passes - no phramacological intervention therapy nor cure is available yet- due to the increasing linear behavior of the propagation. 


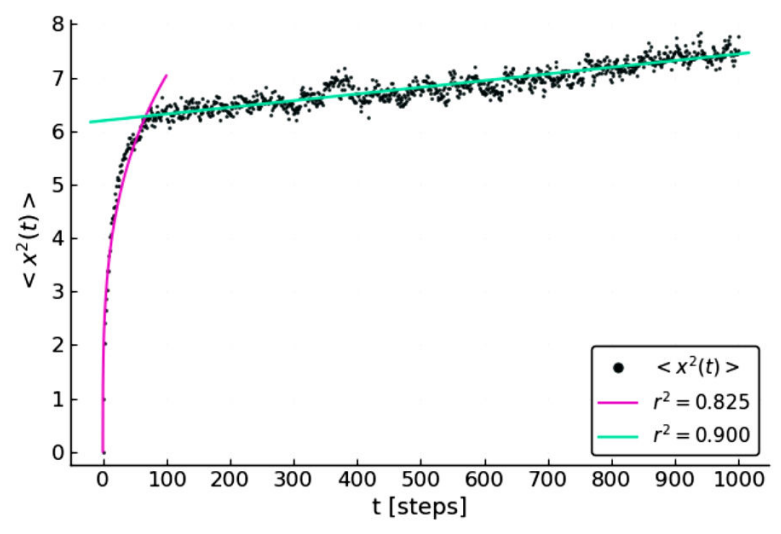

FIGURE 7. Diffusion plot with the mean square displacement as a function of time. The solid line shows the best fit to the data points.

As we mentioned in the introduction, the diffusion modeled by a random walk can be described by Einstein's relation Eq. (8).

In Fig. 7 we plotted the mean square displacement (using Eq. (7)) as a function of time. In this case, we noticed that approximately during the first 100 steps, the behavior is that of an anomalous diffusion, as it is described by a power law with $\alpha=0.289<1$. Thus, the COVID-19 person-toperson airline transportation diffusion -which for the case of this type of disease is the fastest, more effective means of infection nowadays- follows a subdiffusion behavior at first. It then proceeds to take a normal diffusive behavior, with a linearly described mean square displacement.

This behavior indicates how at first the propagation of the coronavirus increases rapidly and after a month it settles to a slightly slower but constant rise of cases. In a similar fashion to what happens with similar viral infections such as SARS, other CoVs and the stationary flu.

\section{Conclusions}

We explored the risk of the COVID-19 virus infection in Mexico using a diffusive model of random walks throughout two different types of airline networks: the Air Transportation Network and Alliances networks seen as multiplex networks, where nodes represent airports, edges represent flights, and each airline is a layer of the multiplex and monoplex networks which stood for single airlines.

The overall infected area of the networks mainly depend on the amount of airports that constitute it. However, Air China's infected area after the simulation is larger, we assume this happens because said airline is the flag carrier of China, having more flights within China [16].

We found the COVID-19's spread behavior is at first that of an anomalous diffusion, specifically a subdiffusion. After approximately one month, the diffusion is overall behaved as a normal one, with a more steady and slower increase.

In particular, Mexico's risk of being infected by the coronavirus increases linearly over time, having an $89.4 \%$ of probability of infection after a year, quite likely an upper bound. So it seems the COVID-19 will eventually and inevitably reach the country, in spite of some travel restrictions that may be imposed. Even if Mexico used to have a relative advantage due to the lack of direct flights between Wuhan and Mexico's airports -that indeed delays the arrival- there are direct flights from already infected countries to Mexico, hence highlighting the networked nature of pandemics. So, as more countries continue to get infected in spite of the Wuhan travel ban, the chances of contracting the disease in Mexico remain high unless some other form of effective medical containment is applied.

The random walk model we used is very simple and works as a first approach to the problem, taking Wuhan as an only starting point (not from other infected countries) and its trajectory. First of all we are just analyzing possible contagions through the Air Tranportation Network, we are not considering other ways of transmission (layers of the multiplex) like the maritime or terrestrial networks. We also do not consider groups of susceptible, infected, and recovered people as in a SIR model $[11,18]$ hence our model is not sensible enough to many situations or changes as other more robust models. Nevertheless, we were able to capture a high risk of infection in Mexico which is what we are in fact witnessing.

As data becomes available it will be possible to fine tune the parameters of the random walk to better reproduce actual contagion. This will allow a simple yet reliable estimator to be used as an auxiliary to survey-based epidemiological reports. Such reports will still be the main source of historical information and the main tool in medical and public health decision making. However, data driven, theoretical approaches such as the one we presented, allow for (inconclusive and preliminary, yet useful) forecasting schemas.

\section{Appendix A.}

On February 28, 2020 Mexico's Health Ministry confirmed its first two cases of the COVID-19 virus [20]. The two men who tested positive for coronavirus recently visited Italy, the country with the highest number of cases outside of Asia at that time.

We looked for the probability of importing the COVID-19 virus through Italy in the first two months of the outbreak.

As of April 1, 2020, Mexico has 1378 confirmed cases either by importation or local transmission. The mexican government publishes a daily updated file with the confirmed cases that Serendipia, a data journalism initiative, publishes in an open format [21].

The file has the following information: Case number, State, Sex, Age, Date of symptom onset, COVID-19 identification, Country of origin, and Arrival date.

With the information provided we calculated the probability Mexico had of importing the SARS-CoV-2 at exactly the date people arrived from the infected countries. The following table presents the number of random walks, out of the 1000 with origin in Wuhan that went through said country's airport and then arrived in Mexico at an exact date. 
TABLE I. COVID-19 importation risk from Italy, the US, Spain, Germany, France, and Singapore at the exact arrival dates of the first confirmed infected people to Mexico.

\begin{tabular}{cccc}
\hline Country of origin & Arrival date & No. random walks & Percentage \\
\hline Italy & February 21 & 16 & $1.6 \%$ \\
United States & February 23 & 51 & $5.1 \%$ \\
Spain & February 28 & 12 & $1.2 \%$ \\
Germany & March 03 & 6 & $0.6 \%$ \\
France & March 06 & 14 & $1.4 \%$ \\
Singapore & March 11 & 11 & $1.1 \%$ \\
\hline
\end{tabular}

\section{Appendix B}

The information of the airports considered infected during the simulation is shown in the table below.

TABLE II. List of infected Cities and airports as of January 26, 2020. IATA codes obtained from Air-Port-Codes [17]

\begin{tabular}{|c|c|c|}
\hline IATA Code & Airport name & City, Country \\
\hline PEK & Beijing Capital International Airport & Beijing, China \\
\hline TSN & Tianjin Binhai International Airport & Tianjin, China \\
\hline TYN & Taiyuan Wusu International Airport & Taiyuan, China \\
\hline CSX & Changsha Huanghua International Airport & Changsha, China \\
\hline KWL & Guilin Liangjiang International Airport & Guilin, China \\
\hline CGO & Zhengzhou Xinzheng International Airport & Zhengzhou, China \\
\hline KMG & Kunming Changshui International Airport & Kunming, China \\
\hline FOC & Fuzhou Changle International Airport & Fuzhou, China \\
\hline TAO & Qingdao Liuting International Airport & Qingdao, China \\
\hline SHA & Shanghai Hongqiao International Airport & Shanghai, China \\
\hline KWE & Guiyang Longdongbao International Airport & Guiyang, China \\
\hline CTU & Chengdu Shuangliu International Airport & Chengdu, China \\
\hline HRB & Harbin Taiping International Airport & Harbin, China \\
\hline MDG & Mudanjiang Hailang Airport & Mudanjiang, China \\
\hline DLC & Dalian Zhoushuizi International Airport & Dalian, China \\
\hline PVG & Shanghai Pudong International Airport & Shanghai, China \\
\hline SYX & Sanya Phoenix International Airport & Hainan Island, China \\
\hline INC & Yinchuan Hedong International Airport & Yinchuan, China \\
\hline HAK & Haikou Meilan International Airport & Haikou, China \\
\hline SHE & Shenyang Taoxian International Airport & Shenyang, China \\
\hline LYA & Luoyang Beijiao Airport & Luoyang, China \\
\hline XUZ & Xuzhou Guanyin International Airport & Xuzhou, China \\
\hline CGQ & Changchun Longjia International Airport & Changchun, China \\
\hline NAY & Beijing Nanyuan Airport & Beijing, China \\
\hline BHY & Beihai Fucheng Airport & Beihai, China \\
\hline WEH & Weihai Dashuibo Airport & Weihai, China \\
\hline WUX & Sunan Shuofang International Airport & Wuxi, China \\
\hline WUS & Nanping Wuyishan Airport & Wuyishan, China \\
\hline JGN & Jiayuguan Airport & Jiayuguan, China \\
\hline WUZ & Wuzhou Changzhoudao Airport & Wuzhou, China \\
\hline
\end{tabular}


TEN

YIC

RIZ

CAN

PKX

$\mathrm{HGH}$

MZG

TPE

NRT

HND

HKG

GMP

ICN

DMK

HKT

BKK

CNX

CEB

HAN

SGN

MFM

KTM

JHB

SIN

JFK

UGN

ORD

SNA

OCW

DFW

SFO

LAX

DCA

SEA

ATL

IAD

MEB

MDW

BOD

LBG

CDG

ORY

MBW

MEL

BWU

SYD
Tongren Fenghuang Airport

Yichun Mingyueshan Airport

Rizhao Shanzihe Airport

Guangzhou Baiyun International Airport

Beijing Daxing International Airport

Hangzhou Xiaoshan International Airport Magong Airport

Taiwan Taoyuan International Airport

Narita International Airport

Tokyo International Airport

Hong Kong International Airport

Gimpo International Airport

Incheon International Airport

Don Mueang International Airport

Phuket International Airport

Suvarnabhumi Airport

Chiang Mai International Airport

Mactan-Cebu International Airport

Noi Bai International Airport

Tan Son Nhat International Airport

Macau International Airport

Tribhuvan International Airport

Senai International Airport

Singapore Changi Airport

John F. Kennedy International Airport

Waukegan National Airport

O'Hare International Airport

John Wayne International Airport

Warren Field Airport

Dallas-Fort Worth International Airport

San Francisco International Airport

Los Angeles International Airport

Ronald Reagan Washington National Airport

Seattle-Tacoma International Airport

Hartsfield-Jackson Atlanta International Airport

Washington Dulles International Airport

Laurinburg-Maxton Airport

Midway International Airport

Bordeaux-Mérignac Airport

Paris-Le Bourget Airport

Paris Charles de Gaulle Airport

Paris Orly Airport

Moorabbin (Harry Hawker) Airport

Melbourne Airport

Melbourne Airport

Sydney (Kingsford Smith) Airport
Tongren, China

Yichun, China

Rizhao, China

Guangzhou, China

Langfang, China

Hangzhou, China

Makung, Taiwan

Taoyuan, Taiwan

Narita, Japan

Tokyo, Japan

Chek Lap Kok, Hong Kong

Seoul, South Korea

Incheon, South Korea

Bangkok, Thailand

Phuket, Thailand

Bangkok, Thailand

Chiang Mai, Thailand

Lapu-Lapu City, Philippines

Hanoi, Vietnam

Ho Chi Minh City, Vietnam

Taipa, Macau

Kathmandu, Nepal

Johor Bahru, Malaysia

Changi, Singapore

New York City, USA

Waukegan, USA

Chicago, USA

Santa Ana, USA

Washington, USA

Dallas and Fort Worth, USA

San Francisco, USA

Los Angeles, USA

Arlington County, USA

Seattle, USA

Atlanta, USA

Washington, USA

Maxton, USA

Chicago, USA

Bordeaux, France

Le Bourget, France

Paris, France

Paris, France

Melbourne, Australia

Melbourne, Australia

Bankstown, Australia

Mascot, Australia 


\section{Appendix C}

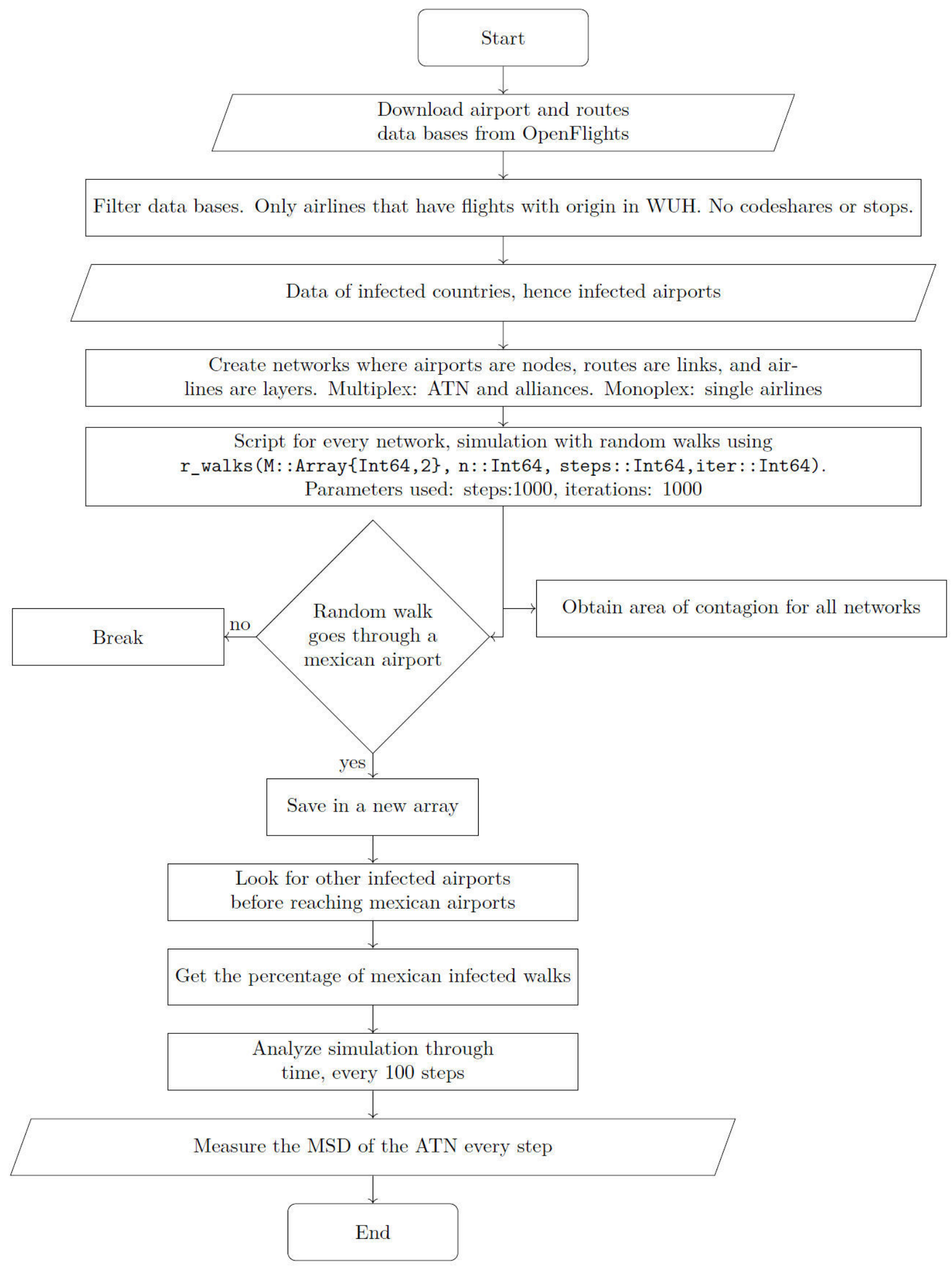

FIGURE 8. Flow chart of the simulation and analysis. 
1. World health organization, "Coronavirus", accessed February 10, (2020). https://www.who.int/health-topics/ coronavirus

2. Healthmap, “COVID-19”, accessed February 14, (2020), https://www.healthmap.org/covid-19/

3. World health organization, "Coronavirus disease (COVID2019) situation reports", accessed April 01, (2020), https://www.who.int/emergencies/diseases/ novel-coronavirus-2019/situation-reports/

4. World health organization, "Statement on the second meeting of the International Health Regulations (2005) Emergency Committee regarding the outbreak of novel coronavirus (2019-nCoV)", accessed February 10, (2020) https://www.who.int/news-room/detail/3001-2020-statement-on-the-secondmeeting-of -the-international-health-regulations-(20 05)-emergency-committee-regarding-the-out break-of-novelcoronavirus-(2019-ncov)

5. World Health Organization, "WHO Director-General's opening remarks at the media briefing on COVID-19 - 11 March 2020", accessed March 11, 2020,

https://www.who.int/dg/speeches/detail/ who-director-general-s-opening-remarks-at -the-media-briefing-on-covid-19-11-march $-2020$

6. World Health Organization, "Coronavirus", accessed February 10, 2020, https://www.who.int/health-topics/ coronavirus/coronavirus\#tab=tab_1.

7. Tokyo 2020, "New dates: 23 July to 8 August 2021", accessed March 30, 2020, https://tokyo2020.org/en/news/ new-dates-tokyo-2020

8. World atlas, "Countries Who Do Not Have An Airport”, accessed September 18, (2019), https://www.worldatlas.com/articles/ countries-who-do-not-have-anairport.html

9. G. N. Cook and J. Goodwin, Airline networks: A comparison of Hub-and-Spoke and Point-to-Point Systems, J. Avi-
ation/Aerospace Edu. Res. 17 (2008) 51. https://doi. org/10.15394/jaaer.2008.1443

10. M. E. O'Kelly, A geographer's analysis of hub-and-spoke networks J. Transp. Geo. 6 (1998) 171. https://doi.org/ $10.1016 / \mathrm{S} 0966-6923(98) 00010-6$.

11. G. Bianconi, Multilayer Networks. (Oxford university press, 2018). 10.1093/0so/9780198753919.001.0001.

12. F. A. Oliveira, R. M. S. Ferreira, L. C. Lapas, and M. H. Vainstein, Anomalous diffusion: A basic mechanism for the evolution of inhomogeneous systems, Front. Phys. 7 (2019) 18. https://doi.org/10.3389/fphy.2019.00018

13. D. Frenkel and B. Smit, Understanding molecular simulation, 2nd ed. (Academic Press, San Diego, 2002), https://doi. org/10.1016/B978-0-12-267351-1.X5000-7

14. Openflights "Airport, airline and route data", accessed April 08, (2019), https://openflights .org/data

15. GitHub, "OpenFlights database", accessed April 08, 2019, https://github.com/jpatokal/openflights/

16. M. Chinazzi et al., The effect of travel restrictions on the spread of the 2019 novel coronavirus (2019-nCoV) outbreak, ePrint: medRxiv: https://doi.org/10.1101/2020. 02.09 .20021261

17. Wikipedia, "Air China", accessed February 26, 2020, https : //en.wikipedia.org/wiki/Air_China

18. M. Newman, Networks, (Oxford university press, 2019).

19. Channel News Asia, "Mexico confirms first two cases of COVID-19, linked to Italy", accessed February 28, 2020, https://www.channelnewsasia.com/news/world/mexicodeclares -first-case-covid-19-coronavirus-health-ministry-12482976

20. Air-port-codes, "Airport codes", accessed February 28, (2020), https://www.air-port-codes.com

21. Serendipia, "Datos abiertos sobre casos de Coronavirus COVID-19 en México", accessed March 20, 2020, https://serendipia.digital/2020/03/ datos-abiertos-sobre-casos-de-coronaviruscovid-19-en-mexico/ 